\title{
Towards a natural system of units for physics and metrology
}

\section{On a re-definition of the base units of the SI, the kilogram, ampere, kelvin and mole, in terms of fundamental physical constants}

\author{
S.G. Karshenboim ${ }^{\mathrm{a}}$ \\ D.I. Mendeleev Institute for Metrology (VNIIM), St. Petersburg 190005, Russia \\ and Max-Planck-Institut für Quantenoptik, 85748 Garching, Germany
}

\begin{abstract}
A few years ago it was proposed by Mills et al., 2005 to redefine the kilogram in terms of values of certain natural constants. It is expected that the consequences of this redefinition will include some disadvantages in mass measurements and advantages in the electric measurements and in some other areas. To achieve the best possible balance between gains and losses it is crucial to create such a version of the SI, in which electric measurements in SI units are possible with the highest accuracy. This can be only done with a simultaneous redefinition of the kilogram and ampere and we consider various details of such a scenario. Further consideration involves a suggestion on a redefinition of the kelvin and mole, which we also consider. Besides, we discuss various general issues of the natural units ranging from fundamental to practical.
\end{abstract}

\section{Introduction}

A recent proposal [1] to redefine the SI kilogram and possibly the ampere by adopting special fixed numerical values of certain fundamental physical constants is considered here. The redefinition would change the International System of units (the SI) [2], which is a commonly accepted coherent system of units for all branches of macroscopic measurements in technology, education and applied sciences and partly in physics.

The further development [3] assumes to redefine not only the kilogram and the ampere, but also suggests to redefine the mole and the kelvin. The redefinition $[1,3]$, which has been proposed in terms of fundamental constants, also indirectly involves certain natural quantum and discrete phenomena, which should appear due to realizations of the suggested definition.

The SI is not a system adopted once and forever, but an evolving system which follows progress in physics and technology. In particular, in 1983 the SI was changed by adopting an exact numerical value of the speed of light in vacuum, $c$ [4], which is in part similar to the suggested change $[1,3]$. However, the present situation is different in a number of crucial aspects. The major problem is that the present high-accuracy measurements in mechanics and electricity are performed in fact in two different versions of the SI. While macroscopic mass measurements are performed in terms of the SI kilogram, the most accurate electric measurements, mainly related to electrotechnics, are performed in terms of the practical units, ohm-90 and volt-90 [5], and their derivatives. The latter are apparently not consistent with the SI.

In electricity both systems of units are sometimes applied simultaneously when calculating the strength of electromagnetic field (e.g., for electric balances) or capacitance (for calculable

\footnotetext{
${ }^{a}$ e-mail: savely.karshenboim@mpq.mpg.de
} 
capacitors). Any calculation of this kind involves the electric or magnetic constants of vacuum, $\epsilon_{0}$ and $\mu_{0}$, numerical values of which are known in the SI units exactly [2]. In other words, as long as we deal with direct measurements only, electricity completely relies on the practical units, but once certain dimensional calculations are involved, electric results are presented in terms of combinations of the SI and practical units related to the same quantity, e.g., to the electric current.

Another field, which massively involves non-SI units, is microscopic mass measurements, related to determination of various masses either in unified atomic mass units or in frequency units, i.e., dealing with $m c^{2} / h$ instead of the mass $m$. Meanwhile, the SI value of the Planck constant $h$ has a larger uncertainty than microscopic mass comparisons, while in practical units-90 the numerical value of $h$ is known exactly. That means that involving $h$ as a conversion factor, the values measured in microscopic units appear to be presented with a high accuracy in units, closely related to the ohm-90 and volt-90. Additionally, the numerical value of the Avogadro constant $N_{A}$, which serves as a conversion factor between the atomic mass unit and the base macroscopic mass unit is also known in practical units substantially better than in SI units.

In this paper we discuss the present status of the problem and consider in detail various issues related to the redefinition both from practical and fundamental points of view.

\section{The SI system and the 1990 practical units}

The proposal [1] has been focussed on the desirability of replacing the definition of the unit for mass measurements, now based on the last artefact of the SI, the kilogram prototype kept at the BIPM in Sèvres near Paris, by a new definition which is stable and independently reproducible. That mainly focussed attention on the kilogram alone, while a redefinition of the ampere was initially considered rather as one of a number of unnecessary collateral options.

On the contrary, we believe that the existing gap between the present version of the SI and the alternative system, based on the ohm-90 and the volt-90, is a crucial reason to consider such a redefinition [6,7]. The contemporary version of the SI [2] was introduced in 1983 by adopting a fixed value of the speed of light $c$ by the International Committee on Weights and Measures (CIPM) [4]. Meanwhile, five years later, in 1988, CIPM recommended a departure from the SI by introducing practical electric units [5] which have been in effect since 1990. The former improved our ability to measure wave lengths in the SI units, while the latter approved the application of certain non-SI units in order to achieve higher accuracies in electric measurements.

The desirability of resolving the inconsistency between the units, used in precision electric and macroscopic mass measurements, and in restoring the SI system as the only system of units for precision macroscopic measurements drives us to a possible redefinition of the kilogram and the ampere at the same time. We remind that the inconsistency in units has appeared because the requirement for performing the most precise electric and mass measurements in the SI units was partly inconsistent by inself. It is still there and will definitely remain there for an uncertain period of time.

The present version of the SI is based on the kilogram prototype and an adopted fixed value of the magnetic constant $\mu_{0}$, while practical units (the units-90) are based on recommended fixed values of the von Klitzing constant

$$
R_{K}=\frac{h}{e^{2}}
$$

and the Josephson constant

$$
K_{J}=\frac{2 e}{h}
$$

The other units are defined in the same way in both systems.

If we intend to construct a certain version of the SI, which allows the derivation of fixed values of $R_{K}$ and $K_{J}$, we have to fix values of two fundamental constants, e.g., the Planck 
constant $h$ and the elementary charge $e$. To fix two values, we must redefine two units at the same time. Any redefinition of the kilogram alone would be of reduced importance. It should be also clear that any redefinition which leaves at least one of the quantum electric constants, $R_{K}$ and $K_{J}$, not known exactly (as, e.g., by adopting exact values for $N_{A}$ and $e$ ) will be also of reduced importance, because the practical electric units will still be necessary and will still differ from the SI units.

We note that once discussions in various commissions reached a practical stage, a redefinition at the same time of the both units, the kilogram and ampere, was supported by consensus, as well as a decision to adopt certain fixed values of $h$ and $e$ for this purpose.

We collect the values of the involved constants in Table 1 . The numerical values are presented there in the SI units and in units-90. The publication of the proposal and the first round of the most intensive discussions (see [7] for detail) took place before 2006 and the most recent CODATA recommended values at that time were the CODATA-2002 values [8]. To avoid unnecessary variety in the data, we also base most of our consideration in this paper and in particular in Table 1 on these values. All further progress in the determination of the values of the involved fundamental constants and related consequences are considered separately sections 7 and 8 .

Table 1. Numerical values of the involved fundamental constants. References: the SI brochure [2], the CODATA-2002 recommended values [8], the practical values adopted by CIPM [5]. $m(\mathcal{K})$ stands for the mass of the international prototype of the kilogram. The units-90 are defined as a system of units, in which $\Omega_{90}, V_{90}, \mathrm{~m}, \mathrm{~s}, \mathrm{~mol}$ and $\mathrm{K}$ are the base units; e.g. $\mathrm{kg}_{90}=\left(V_{90}^{2} / \Omega_{90}\right)\left(\mathrm{s}^{3} / \mathrm{m}^{2}\right)$ etc. $c$ stands for the speed of light in vacuum, since the notation $c_{0}$ for this value is out of use in fundamental physics.

\begin{tabular}{|c|c|c|c|c|c|}
\hline Constant & Expression & Value & Unit & $u_{r}$ & Ref. \\
\hline \multirow[t]{2}{*}{$m(\mathcal{K})$} & & 1 & $\mathrm{~kg}$ & exactly & {$[2]$} \\
\hline & & $1-1.0(1.7) \times 10^{-7}$ & $\mathrm{~kg}_{90}$ & {$\left[1.7 \times 10^{-7}\right]$} & {$[8]$} \\
\hline$c$ & & 299792458 & $\mathrm{~m} / \mathrm{s}$ & exactly & [2] \\
\hline \multirow[t]{2}{*}{$\mu_{0}$} & & $4 \pi \times 10^{-7}$ & $\mathrm{~N} / \mathrm{A}^{2}$ & exactly & {$[2]$} \\
\hline & & $4 \pi \times 10^{-7} \times\left(1-17.4(3.3) \times 10^{-9}\right)$ & $\mathrm{N}_{90} / \mathrm{A}_{90}^{2}$ & {$\left[3.3 \times 10^{-9}\right]$} & {$[8]$} \\
\hline \multirow[t]{2}{*}{$\epsilon_{0}$} & $1 /\left(\mu_{0} c^{2}\right)$ & $8.8541878176 \cdots \times 10^{-12}$ & $\mathrm{~F} / \mathrm{m}^{2}$ & exactly & {$[2]$} \\
\hline & & $\begin{array}{l}8.8541878176 \ldots \\
\quad \times 10^{-12} \times\left(1+17.4(3.3) \times 10^{-9}\right)\end{array}$ & $\mathrm{F}_{90} / \mathrm{m}$ & {$\left[3.3 \times 10^{-9}\right]$} & [8] \\
\hline \multirow[t]{3}{*}{$e$} & & $1.60217653(14) \times 10^{-19}$ & $\mathrm{C}$ & {$\left[1.7 \times 10^{-7}\right]$} & {$[8]$} \\
\hline & & $1.60217649(66) \times 10^{-19}$ & $\mathrm{C}$ & {$\left[4.1 \times 10^{-7}\right]$} & {$[5]$} \\
\hline & & $1.602176492 \cdots \times 10^{-19}$ & $\mathrm{C}_{90}$ & exactly & {$[5]$} \\
\hline \multirow[t]{3}{*}{$h$} & & $6.6260693(11) \times 10^{-34}$ & $\mathrm{Js}$ & {$\left[1.7 \times 10^{-7}\right]$} & {$[8]$} \\
\hline & & $6.6260689(38) \times 10^{-34}$ & $\mathrm{~J} \mathrm{~s}_{\mathrm{s}}$ & {$\left[5.7 \times 10^{-7}\right]$} & {$[5]$} \\
\hline & & $6.626068854 \cdots \times 10^{-34}$ & $\mathrm{~J}_{90} \mathrm{~s}$ & exactly & {$[5]$} \\
\hline \multirow[t]{3}{*}{$R_{K}$} & $h / e^{2}$ & $25812.807449(86)$ & $\Omega$ & {$\left[3.3 \times 10^{-9}\right]$} & {$[8]$} \\
\hline & & $25812.8070(25)$ & $\Omega$ & {$\left[1 \times 10^{-7}\right]$} & {$[5]$} \\
\hline & & 25812.807 & $\Omega_{90}$ & exactly & {$[5]$} \\
\hline \multirow[t]{3}{*}{$K_{J}$} & $2 e / h$ & $483597.879(41) \times 10^{9}$ & $\mathrm{~Hz} / \mathrm{V}$ & {$\left[8.5 \times 10^{-8}\right]$} & {$[8]$} \\
\hline & & $483597.9(2) \times 10^{9}$ & $\mathrm{~Hz} / \mathrm{V}$ & {$\left[4 \times 10^{-7}\right]$} & {$[5]$} \\
\hline & & $483597.9 \times 10^{9}$ & $\mathrm{~Hz} / \mathrm{V}_{90}$ & exactly & {$[5]$} \\
\hline$\alpha$ & $\left(\mu_{0} c\right) /\left(2 R_{K}\right)$ & $137.03599911(46)$ & & {$\left[3.3 \times 10^{-9}\right]$} & {$[8]$} \\
\hline$N_{A}$ & & $6.0221415(10) \times 10^{23}$ & $1 / \mathrm{mol}$ & {$\left[1.7 \times 10^{-7}\right]$} & {$[8]$} \\
\hline$h N_{A}$ & & $3.990312716(27) \times 10^{-10}$ & $\mathrm{~J} \mathrm{~s} / \mathrm{mol}$ & {$\left[6.7 \times 10^{-9}\right]$} & {$[8]$} \\
\hline$k$ & & $1.3806505(24) \times 10^{-23}$ & $\mathrm{~J} / \mathrm{K}$ & {$\left[1.8 \times 10^{-6}\right]$} & {$[8]$} \\
\hline$k / h$ & & $2.0836644(36) \times 10^{10}$ & $\mathrm{~Hz} / \mathrm{K}$ & {$\left[1.7 \times 10^{-6}\right]$} & {$[8]$} \\
\hline
\end{tabular}

Indeed, it is not necessary that, adopting certain fixed values for the Planck constant $h$ and the elementary charge $e$, we will arrive at the units-90. It is rather expected that the adopted values would be somewhat different. However, if we adopt, e.g., $\left\{e_{\text {new }}\right.$ sI $\}=1.00000001 \times\left\{e_{90}\right\}$ and thus $\mathrm{C}_{\text {new } \mathrm{SI}}=(1 / 1.00000001) \times \mathrm{C}_{90}$ etc., that will not touch the accuracy of the numerical 
values and in this sense the units-90 are equivalent to any new SI with fixed values of $h$ and $e$. (The curved parentheses stand here for the numerical values.)

\section{Microscopic and macroscopic quantities}

The separation between the area where we apply the SI and the practical units lies not only between mass measurements and electric measurements, but also between microscopic and macroscopic mass measurements. The latter is not so obvious because it is hidden in the related conversion factors. For instance, we know many particle, nuclear and atomic masses with high accuracy in terms of the proton mass, frequency units (i.e. of $m c^{2} / h$ ) or unified atomic mass units, etc. The conversion factors involve either $h$, or $e$, or both of them. We can successfully translate the result into practical units, in which $h$ and $e$ are known exactly, while translating into SI kilograms we often lose accuracy. More discussion on units for microscopic mass measurements can be found in section 12.3 .

Let us discuss the problem of microscopic and macroscopic quantities in more general terms. Most of the fundamental constants are related to microscopic physics (atomic, nuclear or particle physics) and their numerical values are of two kinds, being a result either of pure microscopic comparison (e.g., the value of $m_{e} / m_{p}$ ) or of comparison between microscopic and macroscopic values (e.g., the value of the electron mass in kilograms or $\mathrm{eV} / \mathrm{c}^{2}$ ).

The pure microscopic data are in general known more accurately (often much more accurately) than the data which involve macroscopic physics. That is mainly a consequence of the limited accuracy of measurements linking macroscopic and atomic physics. Very few really fundamental constants, such as for example the gravitation constant $G$, come from pure macroscopic experiments, but these play rather a marginal role in precision measurements.

Apparently, without new experiments we cannot improve the links between the microscopic and macroscopic physics. However, the numerical values of the fundamental constants play an important role as anchor reference data. Results for X-ray transitions are customarily expressed in units of energy $(\mathrm{eV})$, but not in terms of the frequency or wave length. To interpret a frequency as energy (in eV), one has to apply a certain value of $h / e$. The accuracy of the best comparisons of two transitions is higher than that of the available numerical value of $h / e$ in the SI units [8]. By changing the basis of the definition of the SI units of the mass and the current we can improve quality of the reference data, and the characterization of the X-ray transition in terms of electron-volts would be adequate. This could be achieved by defining the units of the mass and the current, which are now macroscopic, in microscopic terms, i.e., in terms of $h$ and $e$ (for more details see $[6,10]$ and section 12.3 ).

\section{Problem in determination of the Planck constant}

For the SI, the most questionable link between macroscopic and microscopic physics is related to experiments on determination of the Planck constant $h$. There is currently an unresolved discrepancy of $1 \mathrm{ppm}[8,9]$ between the values of the Planck constant derived from the wattbalance experiments and from the X-ray crystal density (XRCD) determination. The results of all other measurements together produce a third value that is competitive in accuracy with the $\mathrm{XRCD}$ result and is in perfect agreement with the watt-balance values. The importance of this third result is often underestimated (see [6,7] for detail). The situation based on CODATA-2002 data [8] is summarized in Fig. 1.

The experiments mentioned above determine a link between the macroscopic mass unit, the kilogram, and the electric power unit, expressed in terms of volt-90 and ohm-90. This is a crucial link for the realization of the SI ampere in the present version of the SI. In the proposed version of the SI [1], based on the kilogram unit defined by an adopted fixed value of the Planck constant $h$ (or, alternatively, of the Avogadro constant $N_{A}$ ), these experiments determine the mass of the kilogram prototype, which is crucial for practical realization of the suggested definition. 


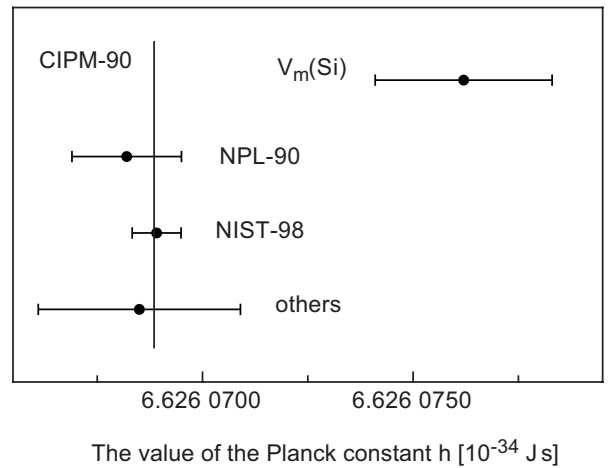

Fig. 1. The determinations of the Planck constant $h$ in [8]. The watt-balance values (NIST-98 and NPL-90) and XRCD result $\left(\mathrm{V}_{\mathrm{m}}(\mathrm{Si})\right)$ are taken directly from [8] and labeled in the same way as there. 'Others' stands for the average values of the rest of the data and was communicated to the author by Peter Mohr on base of [8]. The vertical line indicates a numerical value of $h$ in practical units [5].

Since the publication of the proposal [1], a number of international commissions and committees have considered this issue (see, e.g., [7]). They have emphasized the importance of the problem related to this link and its undesirable effect on accuracy of mass measurements in the case of the redefinition.

Their concerns, however, are based on an assumption that it is up to those, who decide on the redefinition, to involve this link into the SI business or not. We agree that this link is a great problem. But we unfortunately disagree [6,7] that this link can be avoided by, e.g., postponing the redefinition of the kilogram.

This link is crucial in the present-day realization of the ampere (and the volt) of the SI. The electrical units are maintained thought the macroscopic quantum effects and their reproduction is a result of independent determinations of $R_{K}$ and $K_{J}$, or, which is the same, of $h$ and the fine structure constant

$$
\alpha=\frac{e^{2}}{4 \pi \epsilon_{0} h c} .
$$

In other words, the link, based on determination of $h$, has been used since the time, when the Josephson effect and the quantum Hall effect were massively applied to maintain the volt and the ohm, for the realization of a base SI unit, the ampere, and there has been no way to avoid this troubled link.

Due to specific importance of precision determination of the Planck constant $h$ and the fine structure constant $\alpha$, we consider the related recent progress in sections 7 and 8 .

\section{5 “Constant-based" and "artefact-based" units}

We also raise a question about the conceptual difference between a 'constant-based' unit and an 'artefact-based' unit [6]. In the latter case, the definition can have fundamental problems, but it is very instructive - it is clear in a practical sense what the unit is. In most of the measurements (comparisons), the method of the measurement (comparison) is also obviously fixed. There is not much room for any variety in realizing the unit.

In the former case, when a unit is based on a natural constant and certain relations to other quantities (i.e., certain physical laws), there is a number of ways to realize the definition and, as in the case of any scientific experimental activity, the results may disagree. A substantial difference for a constant-based unit and an artefact-based unit is due to possible systematic effects. For the artefact, some reproducible systematic effects may take place. Such reproducibility is an advantage of the artefact from a practical point of view. However, such a systematic effect, being undetectable, can cause a drift of the unit or a reproducible systematic shift. Various differences in possible realizations of the constant-based units may produce a discrepancy but that 
would eventually allow the detection of a possible problem. The very opportunity to discover the problem, even accompanied by possible discrepancies, is an advantage.

In the case of a constant-based unit, systematic effects may be very different. For instance, for determination of the Planck constant, which is the realization of the SI ampere (presently) and of the SI kilogram (in the case of the redefinition), the results come from different branches of physics and disagree with each other. Relative mass measurements and relative electric measurements are more accurate than the link between the mass and the electricity. For the electric units, CIPM has chosen a clear strategy. Conservative results for $h / e^{2}$ and $2 e / h$ (and, consequently, for the present-day realization of the SI volt and ohm) have been accepted [5], while the most accurate measurements are to be performed in the practical units. The same approach should be used for the kilogram in the case of the redefinition.

\section{Discontinuity in units and data and choice of values for the elementary charge $e$ and the Planck constant $h$}

Another feature, different for artefact-based and constant-based units and related standards, corresponds to their discontinuity. When one substitutes one artefact for another, the continuity can be maintained since both artefacts involve residual uncertainties and one can adopt values of their characteristics (the mass, length etc.) in a consistent way. This residual uncertainty cannot be removed or reduced, being in fact the uncertainty of the related definition. With a better understanding of the problem (e.g., of cleaning of the prototype), we can reduce the uncertainty only by adopting a new independent definition and by changing consequently the unit and its material prototype. (The very adoption of the new definition is not so apparent, because such features as the cleaning procedure are not included into the 'official' part of the definition, but certainly they are a hidden part of it.)

In the case of constant-based definitions a certain discontinuity is unavoidable and one can only try to reduce its consequences. For instance, the present SI definition of the ampere suggests that two electric constants, namely $\mu_{0}$ and $R_{K}$, have exact values. That is obvious from the relation

$$
\frac{\mu_{0}}{R_{K}}=\frac{2 \alpha}{c}
$$

where the fine structure constant is a dimensionless constant of Nature, which cannot be changed by a redefinition of the units.

While at present the value of the magnetic constant $\mu_{0}$ is adopted by the definition, the value of the von Klitzing constant is fixed by the same definition to an unknown value, a subject of measurement. The suggested in $[1,3]$ definition adopts under certain conditions a value of $R_{K}$, while the value of the other constant, $\mu_{0}$, becomes a subject of measurements.

We emphasize that each definition fixes in reality both constants exactly, with one of them known and the other to be measured. From the point of view of discontinuity, that means that we substitute one set of exact values by the other and there is no possibility to do such a substitution guessing the proper values to maintain their continuity.

From the practical point of view the discontinuity may be not seen immediately because of limited accuracy in measurements. However, while in the artefact-based scenario such an uncertainty is partly a residual one and cannot be reduced, in the case of a constant-based definition the improvement in accuracy is only a matter of time. In principle, with the suggested definition (if, e.g., one adopts certain values of $e$ and $h$ ) [1], earlier or later the magnetic constant $\mu_{0}$ will be measured with such an accuracy that its departure from the value adopted in the present SI version [2] should be clearly seen.

The CODATA results [8] reflect the best scientific knowledge on the subject to a certain date (the end of 2002), nevertheless, we believe that CIPM should not try to adopt blindly values, the most close to the contemporary CODATA values, assuming that it may allow to avoid any discontinuity. We believe that CIPM should choose a strategy explained in [6] to reduce consequences of the unavoidable discontinuity. 
First, we note that technically we should fix rather constants $K_{J}$ and $R_{K}$ and derive from them related values for $e$ and $h$. Mathematically, there is certainly no difference between choosing values of a pair $K_{J}-R_{K}$ or $e-h$, but physically, since the accuracy in the determination of $K_{J}$ and $R_{K}$ in the SI units is different by an order of magnitude, it is better to deal with $K_{J}-R_{K}$ and not with $e-h$.

Concerning the preferred value of the Josephson constant $K_{J}$, we suggested in [6] to use the CIPM value related to the volt-1990. In other words, to set the new volt of the SI as the volt1990 exactly. The present CODATA value [8] is consistent with that. The discontinuity effect even with CODATA uncertainty [8], which is less conservative than the CIPM uncertainty [5], is below one standard deviation. So, all practical commercial calibrations done in practical volts could be used without any corrections. There will be also no scientific effects since the shift is below the uncertainty.

Concerning the preferred value for $R_{K}$, we note that value of the ohm-1990 is not consistent with the present SI ohm [8]. Adoption of the ohm-1990 as the new ohm of the SI would mean an observable discontinuity. Nevertheless, we believe that its practical effect is quite reduced and suggest to adopt such a definition that the new SI ohm would be equal exactly to the ohm-1990 [6]. There is a number of reasons to expect that the discontinuity effects will be of reduced practical importance.

- Both mentioned versions of the SI are realized in standards. While the ohm-1990 is the maintained resistance unit applied for calibrations, a number of countries have built calculable capacitors to reproduce the farad as defined by the SI. The farad and the ohm are indeed closely related.

- Any accurate calibration of commercial devices has been done up to now in terms of the ohm-1990 and the adoption of the suggested in [6] should provide us with continuity in their calibration.

- Discontinuity in the value of the farad and in other applications related to the calculable capacitors will not take place because the uncertainty in such capacitors is bigger that the change in the unit.

- Discontinuity will definitely take place in reference tables etc.; however, that should happen earlier or later after we change an exact value of $\mu_{0}$ for another. With improvement of accuracy we should see this change. Actually, because of certain inertia in implementation of the definitions, the technical stage can easily take a few years. Because of progress in the determination of $R_{K}$, closely related to the determination of the fine structure constant $\alpha$, we are not granted that accepting a central value of $R_{K}$ from CODATA-2002 adjustment, the CODATA-2006 value will be consistent with that ${ }^{1}$.

- Only a limited number of the reference data will be affected. Much more changes would be related to $K_{J}$ because of massive application of electron-volts in atomic, nuclear and particle physics; however, fortunately, the CIPM-adopted value of $K_{J}$ [5] is consistent with [8].

\section{The most recent CODATA-recommended values (CODATA-2006)}

The first round of the discussion on the proposal [1] took place at various international bodies in 2005 (see [7] for detail). Due to that we have applied in our analysis above the CODATA-2002 values in order to avoid any unnecessary differences in quoted data. We would like to underline that no fundamental changes have happened since that. Still we discuss in this and in the next sections further progress in the determination of the values of $e$ and $h$ and related constants. The correlated determination of $e$ and $h$ can be more clear presented in terms of determination of $\alpha$ and $h$, which we discuss here and in the next section.

\footnotetext{
1 We remind that to adopt an exact value one should deal not with the real CODATA result, which has an uncertainty, but with its central value. Even a good consistency of the data-2002 and the more recent CODATA values, which will not necessarily happen, would only mean that the CODATA results agree within their uncertainties. That does not mean that the 'old' central value related to a less accurate result, should be consistent with the new and more accurate value.
} 


\subsection{Determination of the fine structure constant $\alpha$}

The CODATA 2002 data for the determination of the fine structure constant $\alpha$ [8] involve results obtained by various methods with an accuracy in a broad range. Many of them remain relevant for the CODATA-2006 adjustment (see Fig. 2).

The most accurate value was obtained from the anomalous magnetic moment of electron $a_{e}$ and it dominates in the CODATA-2002 result. It was based on an experiment performed at the University of Washington, as well as the related result in Fig. 2 for [9], which is deduced with help of an improved theory [11] (the theoretical contribution to the uncertainty budget is $u_{r}=2.4 \times 10^{-10}$ against 2002's $\left.u_{r}=9.9 \times 10^{-10}\right)^{2}$. Another measurement of $a_{e}$ was performed at Harvard University with a higher accuracy [12] and now it is responsible in [9] for the dominant value of $\alpha[13]$.

While the most important results of determination of $\alpha$ were in both CODATA adjustments from the study of $a_{e}$, the second values were due to recoil spectroscopy. Similarly to the change in $a_{e}$, we see now [9] two independent experiments. Additionally to the Stanford result from Cs spectroscopy, in the 2006 adjustment a new result from Rb experiment carried out at LKB [14] is added.

The $\alpha$ determination in [9] is summarized in Fig. 2.
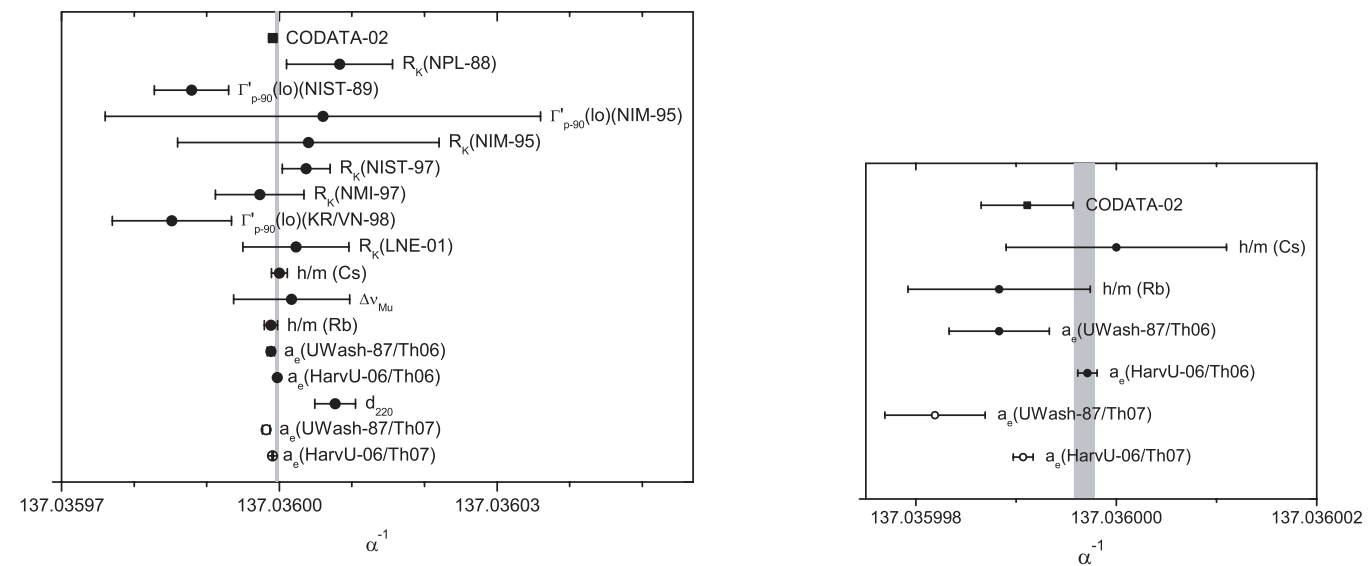

Fig. 2. Determination of the fine structure constant $\alpha$ in the CODATA-2006 adjustment [9] and afterwards. The labels are similar to those in [9]. The vertical band stands for the adjusted CODATA2006 value. The closed circles are for the data included into evaluation [9], while the open circles are for more recent data. The most accurate data from $a_{e}$ and recoil spectroscopy are presented in the right plot with a magnified scale.

\subsection{Determination of the Planck constant $h$}

The determination of the Planck constant $h$ in the 2002 adjustment [8] involved various measurements. Some dealt with electric measurements, some with material. The dominant result was from NIST watt-balance and it is still relevant for 2006 adjustment. Additionally, a new result from NIST [15], which in many details uncorrelated with the older NIST measurement and has a higher accuracy, is included.

The best measurement from XRCD is still in disagreement with the electric measurements (see Fig. 3 for the 2002 adjustment [8] and Fig. 3 for the 2006 adjustment [9]). This problem remains unresolved.

\footnotetext{
${ }^{2}$ Here and throughout the paper we give the references only to the results achieved since the publication of the CODATA-2002 values; for the earlier references see [8].
} 

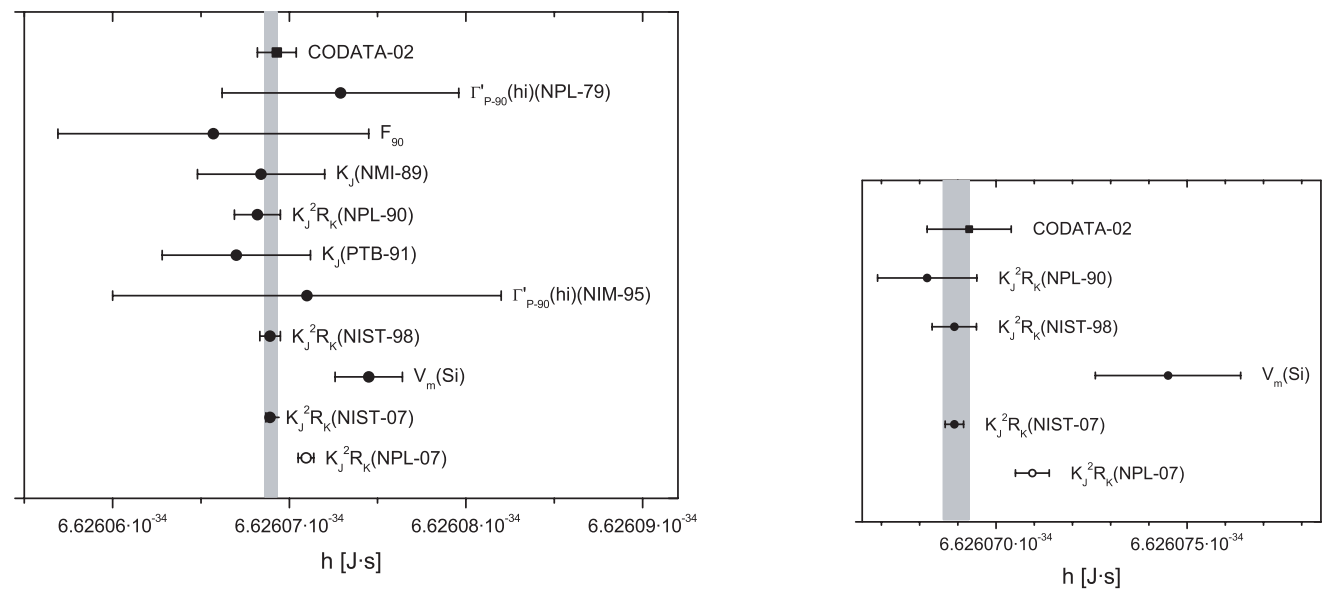

Fig. 3. Determination of the Planck constant $h$ in the CODATA-2006 adjustment [9] and afterwards. The labels are similar to those in [9]. The vertical band stands for the adjusted CODATA-2006 value. The closed circles are for the data included into evaluation [9], while the open circles are for more recent data. The most accurate data from the watt balances and from XRCD measurement are presented in the right plot with a magnified scale.

\section{Progress in determination of values of $\alpha$ and $h$ since CODATA-2006 adjustment}

The CODATA-2006 paper [9] has not yet been published, but certain progress has been already achieved and new data are now available for $\alpha$ and $h$. The related results are also presented in Figs. 2 and 3 with open circles.

\subsection{Determination of the fine structure constant $\alpha$}

Recently an error was discovered in very complicated numerical calculations of the fourthorder contributions to the electron anomalous magnetic moment $a_{e}$ and the related theoretical expression was corrected [16], which leads to a shift in the value of $\alpha$ [17].

\subsection{Determination of the Planck constant $h$}

As we mentioned, the main intrigue in the determination of the values of the constants relevant to the redefinition of the kilogram and other units is a discrepancy between the 'electric' and 'material' values of $h$. A recent watt-balance measurement at NPL [18], published in 2007, delivered a mixed message. We can be more confident for the moment that in general the wattbalance approach is the most accurate and the most reliable method to determine $h$ (until a new-generation XRCD measurement is not performed, which is going to). However, it may look doubtful to trust the accuracy of the watt-balance (until the discrepancy between the recent NIST and NPL results is resolved, or until more watt balances will become operational and they are expected to be).

\section{Looking forward to new results}

First of all, we should explain some time aspects of the redefinition. The crucial time for the redefinition is mid 2009 or 2010. At that time a decision by CIPM is expected. The decision 
should essentially follow a related recommendation by the Consultative Committee on Units (CCU) of CIPM. Eventually, the decision should be approved by the General conference on weights and measures (GCPM) in 2011.

If they fail to redefine, the next window for the redefinition will be in four years. (The four-year period is due to GCPM.)

Usually, in a purely scientific situation, we should consider rather the current status and that allows to estimate consequences. In the problem under discussion, the situation is very different. A number of experiments are of metrological nature; they involve electrical standards or material metrology. The deadlines are due to consideration of various top-level metrological bodies and that makes certain experiments to be of top priority. Due to that it is really expected that a number of experiments (watt-balance determinations of $h$, the XRCD determination of $N_{A}$ and calculable-capacitor determinations of $R_{K}$ ) will be completed by the deadline mentioned. They are launched, encouraged or financed for this purpose.

It is certain that the number of independent results with high accuracy will appear. What is uncertain is whether they will be really consistent. Since the experiments are rather complicated, most of the results will appear in 2009 and 2010 rather than in 2007 and 2008. Their consistency is indeed not granted since we expect a number of really independent experiments applying new methods and a number of experiments which are designed to essentially improve the accuracy of existing methods.

Due to the time limitations and the specifics of the current progress in the field, two crucial moments are most important for the consideration. First, it is important what the situation was at the time when the proposal [1] was launched and massively considered by various international commissions. Second, it is important how it will look like when the decision will be made. The former was basically based on the CODATA-2002 values [8] and we have already discussed that situation above, while the latter should be quite different from the current situation (because of crucial results expected shortly before the coming consideration in 2009 or 2010).

So, we happen to be in a very strange situation: we can observe certain progress, but any analysis of possible consequences based on the current situation is rather of reduced importance. We should wait.

\section{On educational issue due to the SI definitions}

Due to discussion of verbal definitions, the educational side of the problem has been numerously discussed. To our mind, its importance has been overestimated because it is assumed that 'simple people' should understand the definitions. We do not think so. The SI definitions are important in principle for several reasons.

1. The SI system is a system of units and quantities to be a base for legal considerations ${ }^{3}$. The definitions are very important for that; however, we all know that people should understand the laws, but not necessarily know the 'exact' verbal definitions of them. This practice covers all branches of the laws and the result is positive. In this sense, it is necessary for everybody not to know 'exact' definition of the kilogram or the metre, but to understand what each important unit or quantity is.

2. The SI is a base system for a large part of professional education. In the professional education, especially in the natural-science-related education, students should really understand what are the units. However, the world experience suggests a kind of step-bystep education. We are never taught exactly, but we are taught properly, i.e., we obtain the information which is necessary or useful at a certain level of knowledge. A definition can

\footnotetext{
${ }^{3}$ One may say that the metrology has by itself a great practical importance for production, trade, safety etc. via standardization. That is not quite correct. That is the legal system and various supporting bodies that are designed to resolve practical issues, and the metrology has to provide them with a proper instrument for that. With lack of a proper social regulation such as a legal system or its equivalents, the metrological definitions and standards would be of a quite reduced value.
} 
be incomplete, approximate etc. They could be even misleading and 'wrong' in their formal meaning. But that is unimportant. It would be strange to expect, that is is more important for a student to know 'exactly' what the metre is, than to know what an atom is; or whether an atom has a trajectory. It is rather strange to insist that a student should understand what the second is before he could learn the basic quantum mechanics to understand spin and the hyperfine structure etc. Or that the student should learn the definition of the metre before understanding special relativity. We see no reason why the common practice of teaching things step-by-step should be changed for the SI and related matter.

3. There is also a base education which does not have a particular aim, combining 'simplepeople' education for essential life and legal issues, professional pre-education and curiositydriven education. For these cases, no exact definitions are really necessary as well.

The legal aspects of the definitions are the most important. In science and education there are no definitions adopted for universal application. We always may re-phrase a particular definition, or to use an equivalent definition (or an equivalent set of definitions), etc. How to educate students is not a problem of international metrological bodies. All the discussion about the very importance of choosing a proper definition, which should be universally used, is relevant only for the legal use.

\section{On consequences of a redefinition of kelvin and mole by fixing $k$ and $N_{A}$}

While the redefinition of the kilogram and the ampere will have important practical consequences on accuracy of standards, measurements, reference data, introduction practical units etc., the redefinition of the kelvin and mole [3] should rather produce a great methodological impact on the system of physical quantities $[7,19]$.

When we speak about a quantity, we firstly think about a certain general property and next about the information we can extract from the measurement data [20,21] (see discussion below in section 12.3). In principle, the thermodynamic temperature, as a general property, is the average value of the energy per a degree of freedom, while the amount of matter is the number of constituent particles. That is correct as long as we consider them in general. However, information, we can learn from a measurement, depends on units and methods applied.

While the number of particles is a 'countable' value, the amount of matter is a result of weighing a bulk piece of matter (see, e.g., [21] on this issue). For a long time we have been able to weigh more accurately than to count and the results of weighing and counting contained different information because of different accuracy achieved. If we adopt a certain fixed value of the Avogadro constant [3] (independently of the definition of the kilogram), we will change the definition of the mole and the amount of matter. In particular, we will equalize the amount of matter and the number of particles, since the amount of matter will become a counted number of particles. The redefinition will be supported by community if the accuracy of weighing and counting is comparable. That would mean that there should be no reason any longer to distinguish between these two methods and to recognize the results as related to two different quantities.

A similar situation is with the temperature. Once we are able to measure it accurately as the energy, we can determine the Boltzmann constant $k$ with a high accuracy. Adopting a fixed value of $k$, we acknowledge that the temperature is a specific energy and not an independent physical quantity anymore.

Changing definitions of the temperature and the amount of matter will be the first step on a way to reconsider their status as the base SI units (see [7] for details). In the end of this way we see their recognition as specific non-SI units (since we already have SI units for the number of particles and the energy) allowed and may be even recommended for application for historic reasons. But that should take certain time. 


\section{Physical viewpoint on the SI}

\subsection{Physical background of "natural units"}

Still, because of importance of constructing a system of units and quantities for the professional education it is worth considering certain general features of the SI and possible alternative units from the point of view of fundamental physics. Numerous considerations of fundamental constants and natural units were given recently and in the past. For instance, various discussions can be found in [21-23].

Discussing physical meaning of various units in general, we have to consider them at a few levels. First of all, we should stress that there is no just 'system of units'. What we deal with is 'systems of units and quantities'. Comparing the Coulomb law in rationalized and non-rationalized Gaussian systems

$$
\begin{gathered}
\mathbf{F}=-\frac{Q_{1} Q_{2}}{r^{2}} \frac{\mathbf{r}}{r}, \\
\mathbf{F}=-\frac{1}{4 \pi} \frac{Q_{1} Q_{2}}{r^{2}} \frac{\mathbf{r}}{r},
\end{gathered}
$$

we clearly see that the equations suggest different quantities. If, in particular, in both systems the mechanical quantities are defined within the same way, then the charge $Q$ in this pair of equations is defined differently in different lines [19-21]. The 'charges' are different by a factor of $\sqrt{4 \pi}$. Indeed, both 'charges' characterize the same physical property. So, we see one more approach to the quantity as a generic property which allows different 'mathematical' realizations.

Physical consideration judges not about units, but most of all about quantities to describe Nature. The most frequent way of fundamental-physics consideration is a kind of an a priori attempt to describe Nature in the most economic way minimizing a number of quantities needed.

Such a system of quantities could include from one to four base physical quantities, measured in natural units, determined by fundamental constants. The variable number of based units reflects the fact that such quantities as mass, length, frequency and charge are closely related and their interrelations can be seen differently.

\subsection{Fundamental constants and their role for natural units}

To understand why the number of base quantities can vary we should address the issue of fundamental constants. Such constants are the most fundamental parameters of quantum and discrete phenomena, which should be distinguished.

Quantum phenomena are such phenomena, which would be characterized by continuous quantities (in classical physics), but in reality in certain experiments the quantities took only discrete values. The quantization happens because measurements often require eigenvalues of the measured characteristics in the final state. This requirement comes in many situations not only from the measurement details but from the time-evolution, which deals with eigenstates of the Hamilton operator. The values are not necessary quantized in every experiment because some measurements (especially, non-direct measurements) are not related to eigenstates.

Situation with discrete phenomena is not that easy. Some may feel that they are related to classical physics, not quantum. We know that carriers of many quantities such as mass, charge etc. are particles. Since they are of discrete nature they have discrete properties. Three issues here are of quantum nature. First, only in quantum mechanics the particles can be undistinguished and a property such as the electron charge is well defined with absolute accuracy (in classical physics we should expect that it is defined only in average and thus defined approximately). Second, if the 'bricks' are the same, the whole construction is also the same under certain conditions. The structure of compound particles is quite deterministic in quantum mechanics, while in classical physics a construction such as a solar system is peculiar because of accidental 'choice' of initial kinematic parameters and parameters of the 'bricks' (cf. [20]). Thirdly, the quantum field theory suggests as a base way of construction of modern physics, that 
even various parameters of the structureless 'elementary bricks' are not peculiar, but strongly correlated (like the weak interaction coupling constants and $\alpha$, like charge of quarks and leptons etc.). Because of that, such discrete parameters of compound objects like the hyperfine splitting in caesium are well defined, well determined and eventually based on really fundamental quantities. And such elementary parameters as the electron charge and mass are deeply fundamental and are observable by experimental means.

Once we have dimensional parameters we can consider them as fundamental units. Dimensionless parameters and some other dimensional parameters can be considered as conversion factors (see [21] for detail).

In particular, we can consider the speed of light $c$ both: as a fundamental unit of velocity or as a conversion factor between the metre and the second. Here we deal with two separate questions. One is whether the distance and the time intervals are different facets of the same quantity, or these quantities are related but not the same. Most of physicists have their opinion determined, but there is no convincing arguments to support either positions. Both exist; partly that is matter of taste, partly of philosophy.

The other question is whether we should use the same unit for both quantities or not. Technically, that does not depend on our answer on the previous question. However, physically, we should indeed prefer to apply the same unit if be believe that we deal with one quantity and to distinguish the units if we distinguish the quantities.

The same question is related to a pair of the mass (or the energy) and the frequency. That is also to strong extent matter of taste and philosophy (see [20] for detail).

Somewhat different situation is with the electric charge. The majority rather believes that the charge should be expressed in terms of mechanical quantities. The only reason to support it as the fourth base unit suggests that the crucial question is whether the fine structure constant $\alpha$ is calculable or not. If it is not calculable, being a dynamic variable either due to the proportionality of a certain vacuum average, which can take an arbitrary value from a certain range, or because of a connection with a runaway quintessence field, there are certain advantages in considering the charge as an independent base quantity.

\subsection{Quantity and contained information}

Within the SI, in contract to Gaussian units, the current is a base quantity (we could consider the charge as the base electromagnetic quantity, which is physically the same). However, the reason for that is indeed practical, while the 'pure physical' consideration above is in part 'unpractical'. The latter aims rather the best 'theoretical description of the universe', ignoring anything related to the ways to 'experimentally describe the universe', which needs quantities to successfully express the results of measurements for the fundamental phenomena.

We already discussed in section 11 the importance of the information, contained in the numerical values of the quantities. From the general physical point of view, a quantity is first of all a certain generic property of objects or phenomena, while from a more pragmatic view point, which is closely related to metrology, a quantity stands as generic for a set of [numerical] results. The former means that the mass is the same property in any units. The latter clearly distinguishes between the mass $m$ measured in the kilograms and in the unified atomic mass units, because any conversion in either direction suggests a substantial reduction of accuracy. The information contained in $m / \mathrm{kg}$ and $m / \mathrm{u}$ is not the same and thus the values $\mathrm{m} / \mathrm{kg}$ and $m / \mathrm{u}$ describe somewhat different properties (see [10,20] for more discussion).

This difference in understanding of 'quantity' is a quite typical example of application in physics and metrology of the same terms with a related, but somewhat different meaning.

To illustrate the issue of physical contents of the numerical values we summarize in Table 2 various conversion factors for mass related units. The mathematical equations for the conversions are very clear. One can see that the combinations of values such as

$$
m, m c^{2}, m c^{2} / e, m c / h, m c^{2} / h, m / m_{e}, m /\left(m\left({ }^{12} \mathrm{C}\right) / 12\right)
$$

characterize the mass $m$ in one or other way, however in different units $\left(\mathrm{kg}, \mathrm{J}, \mathrm{V}, \mathrm{m}^{-1}, \mathrm{~Hz}\right.$, a.u.(m.), u). 
Table 2. Conversion factors from microscopic mass- and energy-related units into the mass units (the kilogram) in the SI and the units-90. Note: 'a.u.(m.)' stands for the mass in the atomic units $\left(=m_{e}\right)$, to be distinguished from ' $\mathrm{u}$ ' which is for the unified atomic mass unit $\left(=m\left({ }^{12} \mathrm{C}\right) / 12\right)$. The tabulated values are based on [8].

\begin{tabular}{lcc}
\hline Unit & Conversion factor for the SI & Conversion factor for the units-90 \\
\hline $\mathrm{Hz}$ & $7.37249600(37) \times 10^{-51} \mathrm{~kg} / \mathrm{Hz}\left[5 \times 10^{-8}\right]$ & $7.372495882 \cdots \times 10^{-51} \mathrm{~kg}_{90} / \mathrm{Hz}($ exactly) \\
$\mathrm{u}$ & $1.660538782(83) \times 10^{-27} \mathrm{~kg} / \mathrm{u}\left[5 \times 10^{-8}\right]$ & $1.6605387553(23) \times 10^{-27} \mathrm{~kg}_{90} / \mathrm{u}_{90}\left[1.4 \times 10^{-9}\right]$ \\
$\mathrm{eV}$ & $1.782661758(44) \times 10^{-36} \mathrm{~kg} / \mathrm{eV}\left[2.5 \times 10^{-8}\right]$ & $1.782661763 \cdots \times 10^{-36} \mathrm{~kg}_{90} / \mathrm{eV}_{90}($ exactly) \\
$\mathrm{m}^{-1}$ & $2.21021870(11) \times 10^{-42} \mathrm{~kg} \mathrm{~m}\left[5 \times 10^{-8}\right]$ & $2.210218662 \cdots \times 10^{-42} \mathrm{~kg}_{90} \mathrm{~m}($ exactly) \\
a.u. & $9.10938215(45) \times 10^{-31} \mathrm{~kg} /$ a.u. $\left[5 \times 10^{-8}\right]$ & $9.109381999(13) \times 10^{-31} \mathrm{~kg}_{90} / \mathrm{a} . \mathrm{u}\left[1.4 \times 10^{-9}\right]$ \\
\hline
\end{tabular}

In general sense all the quantities listed in (5) characterize the same generic property, namely, the mass. However, we see, that the conversion from various of the applied units into the kilograms has different accuracy in different units within the same system (the SI) and the accuracy is different for the same conversion factor in the SI units and in the units-90. The accuracy of a real measurement could not change because of a change in the units. Switching from the SI to the units-90 we actually change contents of the units and thus of the related numerical values and eventually we relate the data to a different measurement.

The kilogram of the SI is the mass of a specially adopted bulk body, which we can relatively easy compare to the mass of another bulk body. The mass of an electron in terms of the SI characterizes our ability to compare a bulk body and an elementary particle. From all mass-related units listed in Table 2 we can convert the result obtained in them into the SI kilogram with accuracy of 2.5 or 5 parts in $10^{8}$, depending on the unit, while the determination of the electron mass in microscopic units is much more accurate. A relatively low accuracy of the conversion is caused by the fact that all those units are microscopic and hardly linked to any measurements with bulk bodies.

In the units-90 three conversion factors in Table 2 are known exactly and two are improved by more than an order of magnitude. That is because the kilogram has now a microscopic meaning. We have to remind that in the units-90 we still need to measure the mass of macroscopic bodies and its accuracy would be limited in the units-90 by the factor of $5 \times 10^{-8}$.

\subsection{Physical contents of the SI system}

This example stresses a practical issue of metrological approach to units and quantities. Constructing a system of units, we intend to have an instrument appropriate to properly express the results of measurements. Eventually, we need to reach a certain compromise between a simple theoretical description of general properties of Nature and a successful background for expressing the measurement results. That produces 'unnecessary' (from theoretical point of view) units $[20,21]$, which may occupy very different positions within the system of units. For instance, the kelvin and the mole are among base SI units; the unified atomic mass units is outside of the SI, but recommended to be applied along with the SI [2]. There are many units which are not recommended to be used along with the SI; there are also many units, which are used, but often even not officially 'recognized' as units. In particular, the latter is related to natural units such as the nuclear magneton.

Nevertheless, looking from fundamental theoretical perspective, we should not give up at this point and just accept the SI (or another system) as it is formulated. The theoretical understanding of the 'minimal set' of units is still important and relevant. It explains the physical contents of units and physical meaning of quantities (cf. [19]). The fact, that we prefer to characterize the internal energy per a degree of freedom in specific units (the kelvins) cannot change the fact the temperature in the kelvins is just a kind of nickname of the energy per a degree of freedom. 
Discussing the physical meaning, we would like to mention two additional issues. One has to clearly understand that we define quantities prior to the definition of the units and we know a number of their properties independently of the choice of the units. For instance, we know what the mass is and we can judge where the mass of a certain body remains a constant in time independently of units, even if this body is the prototype of the kilogram. The numerical value of its mass is the unity by definition, but its mass rigorously speaking is not a constant and its variation is measurable.

Another related issue is that we should not judge on whether the unit is well or ill defined considering only its definition. It does not matter whether it is written or not, but a part of each definition (sometimes exposed, sometimes hidden) is 'the unit is such a specific value of the quantity, that...'. If we intend to produce a well-defined unit, we should first of all deal with a well-defined quantity. We should be able to do well-defined comparisons in a broad range. We should be able to link the quantity to already defined quantities through natural laws etc. Only after that, to unify the comparisons and applications of the links, and to simplify their interpretation in practice we choose the unit.

In recent years various life- or human-related quantities became important in industry, medicine, safety etc. and they indeed need a strong metrological support. However, one has to remember that such quantities are ill-defined in physical sense and we should distinguish between well-defined physical quantities and some life-related addition. Speaking specifically about the base SI units, we note that the kilogram, metre, second, ampere, kelvin and mole are related to the former, while the candela is for the latter (see [20,21] for more discussion).

\subsection{The SI system and the “new" SI system: Units and fundamental constants}

Changing definition of the base SI units, the kilogram, ampere, kelvin and mole, will change their contents.

First of all, at present the kilogram and ampere are macroscopic units and not well adjusted to measure microscopic and electrotechnical quantities. The status in determination of the values of fundamental constants important for the redefinition of these two units is presented in Fig. 4.

After the redefinition the kilogram and ampere will become microscopic units well adjusted for microscopic mass measurements and for electric measurements, but not for macroscopic mass measurements.

The redefinition will remove $e, h$ and various their derivatives and combinations such as $\hbar$, $K_{J}, R_{K}$ etc. from the list of the adjusted constants since their numerical values will be known
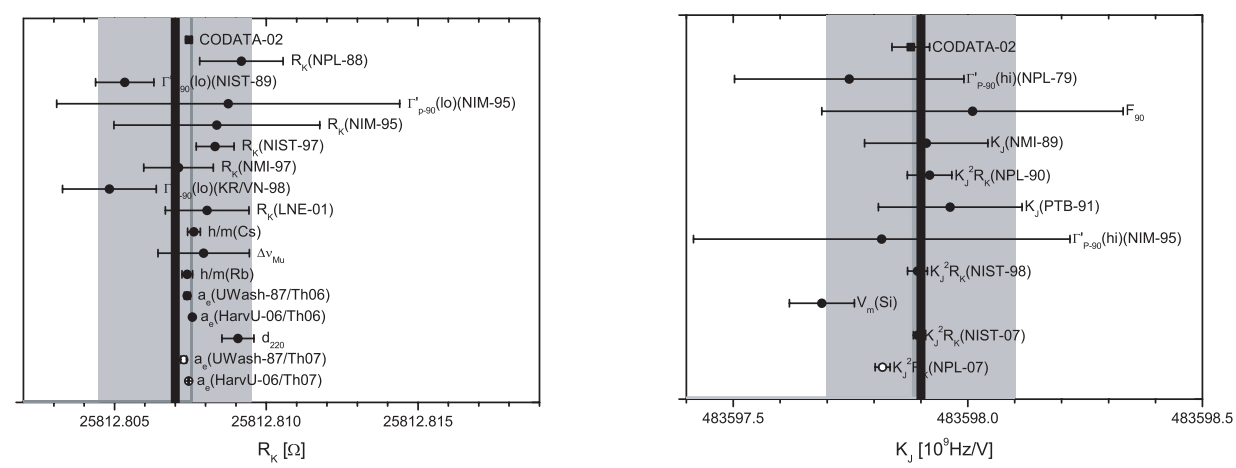

Fig. 4. Determination of the von Klitzing constant $R_{K}$ and the Josephson constant $K_{J}$ in the CODATA-2006 adjustment [9] and afterwards. The labels are similar to those in [9]. The narrow vertical band stands for the adjusted CODATA-2006 value. The closed circles are for the data included into evaluation [9], while the open circles are for more recent data. The broad vertical bands indicate the CIPM recommended values [5] of $R_{K}$ and the Josephson constant $K_{J}$ with lines for the central values suggesting the units-90 are equal to the related SI unit (see Table 1). 


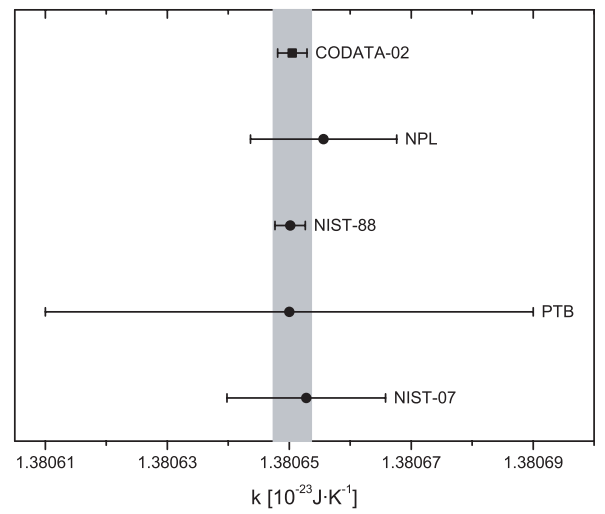

Fig. 5. Determination of the Boltzmann constant $k$ in the CODATA-2006 adjustment [9]. The labels are similar to those in [9]. The vertical band stands for the adjusted CODATA-2006 value.

from the new SI definitions exactly. In return, the electric and magnetic constants of vacuum $\epsilon_{0}$ and $\mu_{0}$ will appear among the adjusted values. The status of the fine structure constant $\alpha$ will indeed not change. The mass of the existing prototype (or an average mass of a certain ensemble of secondary prototypes or another similar value) will be likely adopted as a practical unit and its value $m(\mathcal{K})$ will be for a while an adjustable metrological constant of a great practical importance.

The redefinition of the kelvin and mole has been already discussed in part in section 11 . The present status of determination of $k$ is summarized in Fig. 5. The determination of numerical values of any energy and of $k$ depends indeed on the definition of the kilogram, however, taking into account the accuracy in determination of $k$ and of conversion factor $\mathrm{kg}_{90} / \mathrm{kg}$, which one can indirectly see from Table 2, we see that the redefinition of the kelvin is completely independent of changes in other units.

The triple point of the water will become a measurable quantity of a metrological importance. The Boltzmann constant will indeed disappear from the adjustment. Most of applications will rely on the International Temperature Scale of 1990 (ITS-90) [24] and will not change.

With the adoption of the Avogadro constant the situation is different. The accuracy in determination of $N_{A}$ (after we fix $h$ and $e$ ) will be the same as accuracy for $h N_{A}$ at present. Once we change the contents of the mole, which is for the moment a weighed value, to become a counted value, the Avogadro constant will vanish from the adjustment. Instead of that the carbon molar mass will appear as a measurable quantity.

It is not very clear for a moment where the future CODATA adjustments of the fundamental constants will include $m(\mathcal{K})$, the triple point of the water and the carbon molar mass, since they, being of a great practical importance, are somewhat less fundamental than $h, k$ and $N_{A}$.

\section{Conclusions}

We expect that quite probably the new definition will be adopted in 2011. If that will not happen it is almost certain that they will be adopted in 2015. The redefinition of the four base SI units, namely, the kilogram, ampere, kelvin and mole, will make the SI system more physical. No artefact will be needed and the reproduction of the units will not be related to any particular object.

The next changes in the SI may involve adoption of a new atomic clock for the definition of the second. For decades there was no competition in this area and the caesium clocks have dominated. At present, there are many competitive approaches and some new clocks were considered in the other contributions of this issue. At present the Consultative Committee for Time and Frequency (CCTF) of CIPM forms a list of the most accurately measured atomic transitions reliable for the 'Secondary Representations of the Second'. For the moment these 
transitions are to be used to reproduce the SI second in agreement with the caesium-based definition by applying accurate results on comparison of these transitions against caesium hyperfine transition.

Another change in the SI system, which will hopefully happen in some time, should be a reconsideration of the status of life-related units in the SI. That would be fruitful for physics, since the physical units, such as, e.g. the kilogram, and the life-related units, such as the candela, will be treated differently. That would allow to construct a separate system of physical quantities, well defined, and related physical units, based on fundamental constants. Meanwhile, that would also be productive for the life-related measurements, since all independent liferelated units, such as the candela and the sivert, would be treated in the same manner and the system of life-related quantities and units would have a more logical structure.

The work was supported in part by RFBR grant \#08-02-13516.ofi-z.

\section{References}

1. I.M. Mills, P.J. Mohr, T.J. Quinn, B.N. Taylor, E.R. Williams, Metrologia 42, 71 (2005); B.N. Taylor, P.J. Mohr, Metrologia 36, 63 (1999)

2. The International System of Units (SI), BIPM, Sèvres, 2006; also available on-line at http://www. bipm.org

3. I.M. Mills, P.J. Mohr, T.J. Quinn, B.N. Taylor, E.R. Williams, Metrologia 43, 227 (2006)

4. P. Giacomo, Metrologia 20, 25 (1983)

5. T.J. Quinn, Metrologia 26, 70 (1989); T.J. Quinn, Metrologia 38, 89 (2001); T.J. Quinn, Metrologia 26, 69 (1989)

6. S.G. Karshenboim, On a Natural Definition of the Kilogram and the Ampere: The Objectives and Consequences [physics/0507200]

7. S.G. Karshenboim, Phys.-Usp. 49, 947 (2006)

8. P.J. Mohr, B.N. Taylor, Rev. Mod. Phys. 77, 1 (2005)

9. P.J. Mohr, B.N. Taylor, D.B. Newell, Rev. Mod. Phys. 80, 633 (2008); P.J. Mohr, B.N. Taylor, D.B. Newell, Phys. Today 60, 52 (2007) and at http://physics.nist.gov/cuu/Constants/index.html

10. S.G. Karshenboim, in Precision Physics of Simple Atoms and Molecules, edited by S.G. Karshenboim (Springer, Berlin, Heidelberg, 2007), p. 35

11. T. Kinoshita, M. Nio, Phys. Rev. D 73, 013003 (2006)

12. B. Odom, D. Hanneke, B. D’Urso, G. Gabrielse, Phys. Rev. Lett. 97, 030801 (2006)

13. G. Gabrielse, D. Hanneke, T. Kinoshita, M. Nio, B. Odom, Phys. Rev. Lett. 99, 030802 (2006)

14. P. Cladé, E. de Mirandes, M. Cadoret, S. Guellati-Khélifa, C. Schwob, F. Nez, L. Julien, F. Biraben, Phys. Rev. Lett. 96, 033001 (2006)

15. R.L. Steiner, E.R. Williams, D.B. Newell, R. Liu, Metrologia 42, 431 (2005); R.L. Steiner, E.R. Williams, R. Liu, D.B. Newell, IEEE Trans. 56, 592 (2007)

16. T. Aoyama, M. Hayakawa, T. Kinoshita, M. Nio, Phys. Rev. Lett. 99, 110406 (2007)

17. G. Gabrielse, D. Hanneke, T. Kinoshita, M. Nio, B. Odom, Phys. Rev. Lett. 99, 039902 (2007)

18. I.A. Robinson, B.P. Kibble, Metrologia 44, 427 (2007)

19. S.G. Karshenboim, E.Yu. Korzinin, IEEE Trans. 56, 444 (2007)

20. S.G. Karshenboim, Can. J. Phys. 83, 767 (2005)

21. S.G. Karshenboim, Phys.-Usp. 48, 255 (2005)

22. L.B. Okun, Nucl. Phys. Proc. Suppl. 110, 151 (2002); M.J. Duff, L.B. Okun, G. Veneziano, J. High En. Phys. 3, 023 (2002); G. Fiorentini, L. Okun, M. Vysotsky, JETP Lett. 76, 485 (2002); L.B. Okun, in Astrophysics, Clocks and Fundamental Constants, edited by S.G. Karshenboim, E. Peik (Springer-Verlag, Berlin, Heidelberg, 2004), p. 57; L.B. Okun [physics/0407099]

23. C.J. Bordé, Phil. Trans. Royal Soc. A 363, 2177 (2005)

24. H. Preston-Thomas, Metrologia 27, 3 (1990); Erratum: Metrologia, 107 